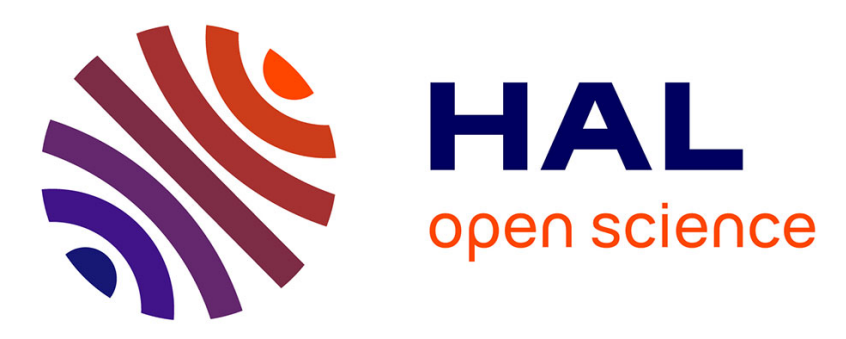

\title{
Entropy-complexity analysis in some globally-coupled systems
}

Antoine M. Chrisment, Marie-Christine Firpo

\section{To cite this version:}

Antoine M. Chrisment, Marie-Christine Firpo. Entropy-complexity analysis in some globally-coupled systems. Physica A: Statistical Mechanics and its Applications, 2016, 10.1016/j.physa.2016.05.009 . hal-01238068v2

\section{HAL Id: hal-01238068 \\ https://hal.science/hal-01238068v2}

Submitted on 16 Jun 2016

HAL is a multi-disciplinary open access archive for the deposit and dissemination of scientific research documents, whether they are published or not. The documents may come from teaching and research institutions in France or abroad, or from public or private research centers.
L'archive ouverte pluridisciplinaire HAL, est destinée au dépôt et à la diffusion de documents scientifiques de niveau recherche, publiés ou non, émanant des établissements d'enseignement et de recherche français ou étrangers, des laboratoires publics ou privés. 


\title{
Entropy-complexity analysis in some globally-coupled systems
}

\author{
Antoine Chrisment ${ }^{\mathrm{a}}$, Marie-Christine Firpo ${ }^{\mathrm{a}, *}$ \\ ${ }^{a}$ Laboratoire de Physique des Plasmas, CNRS-Ecole Polytechnique, 91128 Palaiseau cedex, France
}

\begin{abstract}
Globally-coupled $N$-body systems are well-known to possess an intricate dynamics. When $N$ is large, collective effects may drastically lower the effective dimension of the dynamics breaking the conditions on ergodicity necessary for the applicability of statistical mechanics. These problems are here illustrated and discussed through an entropy-complexity analysis of the repulsive Hamiltonian mean-field model. Using a Poincaré's section of the mean-field time series provides a natural sampling time in the entropy-complexity treatment. This approach is shown to single-out the out-of-equilibrium dynamical features and to uncover a transition of the system dynamics from low-energy non-Boltzmann quasi-stationary states to high-energy stochastic-like behavior.
\end{abstract}

Keywords:

Deterministic chaos, stochasticity, long-range interacting systems, mean-field models, quasi-stationary states

\section{Introduction}

In a seminal work [37, 29], Wold established that any stationary process can be decomposed into the sum of a purely random and a purely deterministic process. Accompanying the modern construction of the theory of chaos has then emerged the quest for dynamical indicators that may discriminate between noise and deterministic chaos and may quantify the respective fractions of these. Given an experimental signal, one would typically like to know whether it is either of deterministic origin, being regular or chaotic, or of random nature, either partly or completely. In this line of research, a significant contribution came from the introduction of a measure of complexity for time series $[3,16]$. Recently, Rosso et al. introduced a representation space, the entropy-complexity causality plane [28], as a novel tool to analyze the chaotic and/or stochastic nature of dynamical systems. Testing this frame against well-known chaotic maps and stochastic processes, this work gave evidence that the entropy-complexity plane could offer a visual representation of the respective weights of the chaotic and stochastic (noise) components of time signals. Nevertheless the contours of the dynamical information devised under the term of 'complexity' (see e.g. the book [2] for an introduction to this concept) remain a subject of active research as well as the conditions of applicability of the entropy-complexity plane to continuous time signals instead of maps [7].

\footnotetext{
*Corresponding author

Email address: marie-christine.firpo@lpp.polytechnique.fr (Marie-Christine Firpo )
} Preprint submitted to Physica A 
In this study, it is proposed to consider the entropy-complexity frame as a new complementary tool to analyze the dynamics and transport properties of $N$-body systems. We have in mind longrange systems, and in particular mean-field systems, that are well-known to exhibit both special equilibrium properties (e.g. with the possibility of ensemble inequivalence [18]) and relaxation properties $[5,14,35]$ such as long-lived out-of-equilibrium states supported by collective waves or other quasi-stationary states. These are ergodicity-breaking features due to low collisionality impeding the relaxation towards Gibbs-Maxwell equilibrium and/or due to possibly insufficient intrinsic stochastic properties. Therefore these systems have a central role to play in the identification of the dynamical requirements for the validity and extensions of statistical mechanics $[33,34,10]$. Simultaneously, another objective of this study is also to test the entropy-complexity frame through its application to the HMF model, that is a more involved system than maps and a time-continuous system, contrarily to maps, but that is a more definite and well-controlled system than truly experimental signals, for which the entropy-complexity frame has started to be used with a vivid interest e.g. in plasma physics $[21,13,36]$.

Because much of the information on the dynamics of $N$-body globally-coupled systems is usually contained in the time evolution of some low-dimensional subset of collective macroscopic variables, it is meaningful to focus on the characterization of the chaotic or stochastic properties of these few relevant collective variables. A prototypical example of such systems is the well-known Hamiltonian Mean Field (HMF) model [1] that will be introduced in Section 2. If the all-to-all particle coupling is repulsive, the HMF system was shown to exhibit some puzzling out-of-equilibrium dynamics in the low energy regime with the emergence of long-lived bicluster patterns whereas the equilibrium statistical mechanics predicts an homogeneous phase for all energies [6]. This transition will serve here to probe the entropy-complexity analysis. This will be introduced and discussed in Section 3. Then, a first dynamical signature of the transition between the very low-energy inhomogeneous states and upper-energy homogeneous ideal gas-like states will be exhibited in Section 4 through some continuous trace in the entropy-complexity plane. As a byproduct of this study, it will be emphasized that, in the large energy limit, the purely deterministic, and here almost non-chaotic ${ }^{1}$, HMF model produces a stochastic-like time behavior of the mean-field. This provides an illustration that time series having stochastic features can emanate from deterministic systems, which puts some limit on the possibilities of discriminating between the noisy or deterministic character of the governing dynamics. In Section 5, the issue of the influence of the sampling period in time-continuous systems on the estimation of the entropy and complexity indicators is addressed. Using a Poincaré's section approach, by considering the time series of the relative maxima of the mean-field, is shown to enable to single-out in the entropy-complexity plane the low-energy regime where dynamical anomalies take place. A short conclusion evoking the potential applications and perspectives of this work is given in Section 6 .

\section{Dynamics of the globally-coupled repulsive HMF model}

A paradigmatic conservative globally-coupled system is the so-called Hamiltonian Mean Field (HMF) model where $N$ particles are moving on a circle being globally coupled by a cosine

\footnotetext{
${ }^{1}$ As long as the number of particles, $N$, in the HMF model is finite, the maximal Lyapunov exponent should be strictly positive, yet scaling as $N^{-1 / 3}[11]$.
} 
interaction with trajectories deriving from

$$
H(\mathbf{p}, \mathbf{q})=\sum_{i=1}^{N} \frac{p_{i}^{2}}{2}+\frac{c}{2 N} \sum_{i, j=1}^{N}\left[1-\cos \left(q_{i}-q_{j}\right)\right] .
$$

The interacting potential is of the same form as in the non-conservative Kuramoto model and both systems bear similarities. For instance, a puzzling $N^{1.7}$ scaling of the lifetimes of homogeneous quasistationary states was reported for the attractive HMF model [38] strangely resonating with the $N^{-1.69}$ scaling of the diffusion coefficient reported in chimera states of the Kuramoto model [26, 25]. An explanation for this strange scaling was proposed in Ref. [9] in the HMF frame on the basis of a stochastic, diffusive, approach.
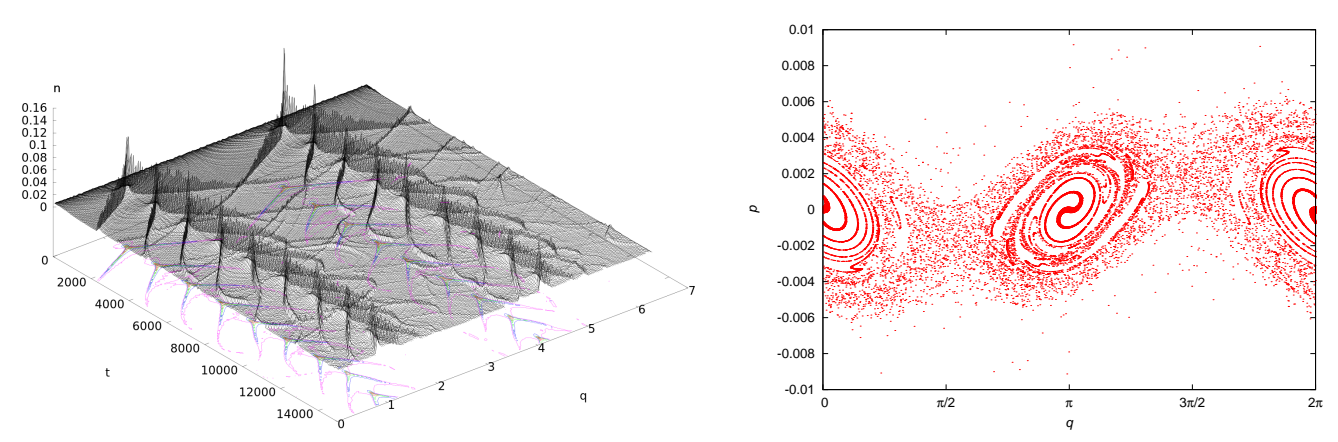

Figure 1: (left) Early time evolution of the density of the $N$ particles on the $\mathbb{R} / 2 \pi \mathbb{Z}$ circle and (right) snapshot of the one-particle $(q, p)$ space at time $t=30000$ for the energy density $\varepsilon=H / N=-0.4999$. In these simulations, $N=20000$ particles were used.

Introducing the collective variable usually called the magnetization, using an analogy of the HMF potential with the X-Y spin model, defined by

$$
\mathbf{M}=\left(M_{x}, M_{y}\right)=\left(\frac{1}{N} \sum_{i=1}^{N} \cos q_{i}, \frac{1}{N} \sum_{i=1}^{N} \sin q_{i}\right),
$$

the equation of motion of any particle $i$ may be written

$$
\frac{d^{2} q_{i}}{d t^{2}}=-c M_{x}(t) \sin q_{i}+c M_{y}(t) \cos q_{i}
$$

For a negative coupling constant $c$, the equilibrium statistical mechanics of the HMF model predicts a vanishing canonical ensemble average of the modulus $M$ of the mean-field. Moreover, the equivalence of the canonical and microcanonical ensembles has been proved for this system [5]. Therefore, from an equilibrium statistical mechanics point of view, the repulsive HMF model appears as trivial, and consequently, uninteresting. However, the numerical symplectic computations of its dynamics revealed some puzzling out-of-equilibrium features. Putting the constant $c$ equal to -1 , the minimal accessible energy density is $\varepsilon=H / N=-1 / 2$. For very low energy simulations, when $\varepsilon$ approaches $-1 / 2$, a very robust biclustered state was observed to form on the $\mathbb{R} / 2 \pi \mathbb{Z}$ circle, the initiation of which is represented on Figure 1. 


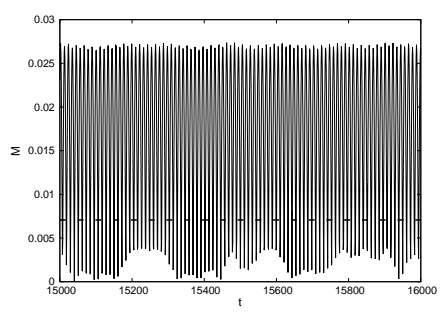

(a) $\varepsilon=-0.498$

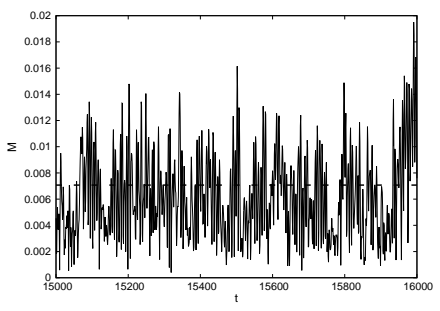

(b) $\varepsilon=0.06$

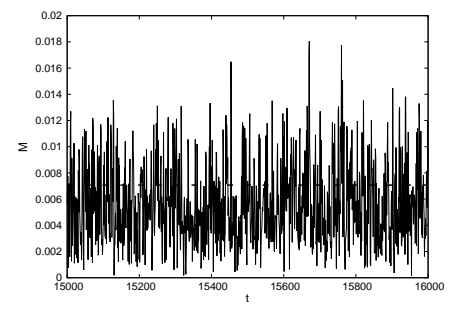

(c) $\varepsilon=5.75$

Figure 2: Parts of some time series of $M(t)$ at different energy densities with $N=20000$ particles. The dashed lines mark the finite- $N$ noise fluctuation level, $1 / \sqrt{N}$, for the magnetization.

This phenomenon was mostly elucidated as a nonlinear collective effect and an effective picture of the quasistationary biclustered states was proposed in Refs. $[4,17]$. In the present study, our objective is to use time series of the mean-field modulus $M(t)=\left(M_{x}^{2}+M_{y}^{2}\right)^{1 / 2}$, a collective observable, in the quasisteady states at different energy densities, to probe the entropy-complexity analysis and extract information on the nature of the dynamics from it. Some parts of the time series used are represented on Figure 2. These numerical results have been obtained using a fourth-order symplectic integrator [39] using a time-step $\Delta \tau=10^{-2}$ ensuring notably a very robust conservation of the total energy. Apart from the results presented in Section 5, our initial conditions were equispaced positions $q_{i}$ and a sinusoidal amplitude velocity perturbation with the $p_{i}$ 's proportional to $\sin q_{i}$.

Finally, let us make an important point. From Poincaré's recurrence theorem, it follows that, in the volume-preserving, and thus Hamiltonian, context, almost any point is recurrent. This a priori prevents the existence of genuine, possibly low-dimensional, chaotic attractors that contrarily may exist in dissipative systems. It is therefore not a priori possible to reconstruct the phase space of a $N \gg 1$-body Hamiltonian system with a low embedding dimension. However, the HMF model considered here is a globally-coupled, mean-field system. Discarding the self-consistency, needed to compute the time behavior of $\mathbf{M}(t)$ in Eq. (3), this $N$-body model would amount to just a one-and-a-half degrees of freedom Hamiltonian, or more exactly, a collection of $N$ uncoupled one-and-a-half degrees of freedom Hamiltonian systems with

$$
H=\sum_{i=1}^{N}\left(\frac{p_{i}^{2}}{2}+M_{x}(t) \cos q_{i}+M_{y}(t) \sin q_{i}\right) .
$$

Therefore the test-particle one-and-a-half degrees of freedom Hamiltonian contains already much information on the full $N$ degrees of freedom HMF model. In particular, the one-and-a-half degrees of freedom systems are known to possess a divided phase space, in some parameter range, with islands of integrability surrounded by a stochastic sea. This striking property explains why mean-field models like the HMF model may have an effective low-dimensional dynamics: clusters of particles within islands may be treated as a few macroparticles, whereas the stochastic sea may be captured with some few other collective degrees of freedom. For instance, Tennyson, Meiss and Morrison [32] considered a mean-field system, close to the HMF model, observed it to relax into a time asymptotic periodic state where only a few collective degrees were active and constructed its low dimensional reduction.

All this leads us to conjecture that the HMF model can display some low dimensional dynamic 
properties that may be captured within a complexity-entropy approach, that is introduced in the following Section.

\section{The entropy-complexity approach}

\subsection{Presentation}

In order to incorporate the intuitive notion of complexity of a physical system, in relation with the amount of order/disorder present in its states, and the hierarchy between them, a statistical measure of complexity was proposed in terms of access probabilities [19]. Its construction started from the assessment that two opposite fundamental $N$-body systems could be viewed as being simple, and be defined as two limits of zero-complexity, namely: i) the perfect crystal and ii) the isolated ideal gas. The perfect crystal is completely ordered and therefore a state is privileged : the hierarchy between accessible states is strong, such that, for this system, the distance to the equiprobable distribution is maximal. Conversely, the isolated ideal gas is completely disordered and all its microstates are equiprobable.

These limit-zero complexity states led to the introduction of the notion of disequilibrium: Whereas entropy measures the degree of disorder, disequilibrium is defined to be the distance between the equiprobable distribution and the distribution of microstates of the studied system, such that complexity is the product of those two measures

$$
[\text { Complexity }]=[\text { Disequilibrium }] \times[\text { Entropy }] \text {. }
$$

A measure of complexity [16] is also expected to satisfy the following requirements: it should be intensive, be able to distinguish among different degrees of periodicity and it should give the most exactly possible an indication of the nature (chaotic or integrable) of the dynamics. From those constraints, a relevant choice made by many authors [28, 24] has been to choose in the complexity definition's (5) the Jensen-Shannon disequilibrium and the Bandt-Pompe's permutation entropy, the definitions of which will be recalled in the following.

Let us start to introduce the Bandt-Pompe's permutation entropy. Our working objects are time series from some physical observable. Given a time series, one examines a sequence, chosen randomly, of $d$ successive points.

Here $d$ denotes the so-called embedding dimension, chosen to be rather small [28]. In particular, Bandt and Pompe suggest for practical purposes working with $3 \leq d \leq 7$ [3], and in the present work we shall use $d=5$ (while e.g. Rosso et al. use $d=6$ in [28]).

Then one asks: What is the permutation of those $d$ points which sorts them by increasing order? The occurrence probability $\mu_{i}(i \in\{1, \ldots, d !\})$ of a permutation $\pi_{i}$ is defined as being the probability that the answer to the later question is $\pi_{i}$. Then the permutation distribution of the time series is defined through the vector of $[0 ; 1]^{d !}$

$$
\mathbf{P}=\left(\mu_{1}, \ldots, \mu_{d !}\right) \text { with } \sum_{i=1}^{d !} \mu_{i}=1
$$

Therefore the uniform permutation probability distribution $\mathbf{P}_{e}$ corresponds to $\mu_{i}=\frac{1}{d !}, i \in$ $\{1, \ldots, d$ ! $\}$. Let $\Omega_{d}$ be the set of permutation probability distributions of size $d$. 
Definition 3.1 (Bandt-Pompe's permutation entropy). The Bandt-Pompe's permutation entropy, $S_{B P}$, [3] of order $d$ is the Shannon entropy, $S_{S}$, of a $d$-order permutation probability distribution

$$
\forall \mathbf{P} \in \Omega_{d}, \quad S_{B P}(\mathbf{P})=S_{S}(\mathbf{P})=-\sum_{i=1}^{d !} \mu_{i} \log \mu_{i}
$$

and therefore the Bandt-Pompe's (permutation) entropy is the restriction of the Shannon's one on $\Omega_{d}$.

Consequently, the permutation probability distribution which maximizes the Bandt-Pompe entropy is the uniform one, $\mathbf{P}_{e}$, corresponding to a time series of the highest degree of randomness

$$
\max _{\mathbf{P} \in \Omega_{d}} S_{B P}(\mathbf{P})=S_{B P}\left(\mathbf{P}_{e}\right)=\log (d !)
$$

The permutations distribution which minimize the Bandt-Pompe entropy are those for which $(d !-1)$ occurrence probabilities are equal to 0 (so one of them is equal to 1 ), corresponding to monotonic time series, so that

$$
\min _{\mathbf{P} \in \Omega_{d}} S_{B P}(\mathbf{P})=0-1 \log 1=0 .
$$

The disequilibrium, noted $Q$, is proportional to the distance between the probability distribution $\mathbf{P}$ and the uniform one : $Q(\mathbf{P})=\eta D\left(\mathbf{P}, \mathbf{P}_{e}\right)$, where $\eta \in \mathbb{R}_{+}^{*}$ is such that $0 \leq Q \leq 1$. The choice of $D$ is crucial and in this study, according to constraints evoked before, the measure of the disequilibrium has consisted of using a Jensen-Shannon divergence defined by [16]

$$
\forall \mathbf{P}_{1}, \mathbf{P}_{2} \in \Omega_{d}, J\left(\mathbf{P}_{1}, \mathbf{P}_{2}\right)=S_{S}\left(\frac{1}{2} \mathbf{P}_{1}+\frac{1}{2} \mathbf{P}_{2}\right)-\frac{1}{2} S_{S}\left(\mathbf{P}_{1}\right)-\frac{1}{2} S_{S}\left(\mathbf{P}_{2}\right)
$$

such that the distance is $D\left(\mathbf{P}, \mathbf{P}_{e}\right)=J\left(\mathbf{P}, \mathbf{P}_{e}\right)$. It remains then to estimate $\eta$. As $Q$ measures a deviation from the uniform distribution, $Q$ is maximal for distributions for which $(d !-1)$ occurrence probabilities are equal to 0 , so that one of them is equal to 1 . Designing by $\mathbf{P}_{m}=$ $(0, \ldots 0,1,0, \ldots, 0)$ one of such a distribution (the 1 is at the $m$ th position), from

$$
\frac{1}{2} \mathbf{P}_{m}+\frac{1}{2} \mathbf{P}_{e}=\left(\frac{1}{2 d !}, \ldots \frac{1}{2 d !}, \frac{1+\frac{1}{d !}}{2}, \frac{1}{2 d !}, \ldots, \frac{1}{2 d !}\right),
$$

one deduces $S_{S}\left(\frac{1}{2} \mathbf{P}+\frac{1}{2} \mathbf{P}_{e}\right)$, and, because $S_{S}\left(\mathbf{P}_{m}\right)=0$ and $S_{S}\left(\mathbf{P}_{e}\right)=\log (d !)$, one obtains

$$
\begin{aligned}
& 1=\max _{\mathbf{P} \in \Omega_{d}} Q(\mathbf{P})=Q\left(\mathbf{P}_{m}\right) \\
&=-\frac{\eta}{2}\left(\frac{d !+1}{d !} \log (d !+1)-2 \log (2 d !)+\log (d !)\right) .
\end{aligned}
$$

Consequently, the Jensen-Shannon's statistical disequilibrium of any probability distribution $\mathbf{P} \in$ $\Omega_{d}$ reads

$$
Q_{J S}(\mathbf{P})=\frac{S_{S}\left(\frac{1}{2} \mathbf{P}+\frac{1}{2} \mathbf{P}_{e}\right)-\frac{1}{2} S_{S}(\mathbf{P})-\frac{1}{2} S_{S}\left(\mathbf{P}_{e}\right)}{-\frac{1}{2}\left(\frac{d !+1}{d !} \log (d !+1)-2 \log (2 d !)+\log (d !)\right)},
$$

where the $J S$ subscript has been added. From this follows, the definition of the complexity used in our study. 
Definition 3.2 (Jensen-Shannon's statistical complexity). The Jensen-Shannon's statistical complexity is defined by

$$
C_{J S}=Q_{J S} \times s_{B P} \quad \text { where } \quad s_{B P}=\frac{S_{B P}}{\log (d !)},
$$

where $Q_{J S}$ is given in Eq. (13).

\subsection{Some reference tests}

In Ref. [28], the entropy-complexity (CS) frame was tested against some time series given, in particular, by some well-known maps and stochastic processes. It was then shown to accommodate noise and chaos at different planar locations, stochastic processes occupying roughly speaking the middle complexity zone of the right corner of the plane up to the white noise limit of normalized entropy equal to one and zero complexity, whereas the most chaotic maps locate mostly in the upper complexity zone. We also performed some 'benchmark' of the CS frame using maps. In Figure 3, the results of the entropy complexity analysis, with $d=5$, of three Schuster maps have been plotted. The Schuster maps are defined by $x_{n+1}=x_{n}+x_{n}^{z} \bmod 1$. Maps are however somehow artificial time series in the sense that time is intrinsically discretized, so that the question of the sampling precision is irrelevant.

In the CS-frame, time-periodic physical signals should be of zero-complexity. Yet, this limit is only theoretical in the sense that the unavoidable discretization prevents this limit from being reached. In order to precise this point, we used time series of some sine functions $\sin (\omega t)$ with various angular frequencies $\omega$, having thus frequencies $f=\omega /(2 \pi)$. In this process, we chose $\omega$ between 1 and $10^{4}$ while the sampling frequency was kept constant and equal to $f_{s}=100$.

The positions in the CS frame of these time series are very instructive and represented on Figure 3. As expected from the entropy-complexity analysis, the different degrees of periodicity are distinguished. When the sine function is the most finely sampled, i.e. in the small $f / f_{s}$ limit, the complexity and entropy of the associated time series are minimal. Conversely, it is not surprising to see that among the various sine functions tested, both the maximal complexity and the maximal entropy are obtained for the case when the sine frequency $f$ becomes of the same order as $f_{s}$. Indeed, according to Shannon's theorem [30], the minimal sampling frequency enabling to reconstruct a signal of frequency $f$ from its time series is $2 f$. Moreover, when $2 f$ becomes larger than $f_{s}$, there is the well-known effect of spectral aliasing: a stroboscopic effect comes into play that makes a high frequency signal have an apparent frequency lower than $f_{s} / 2$. Additionally, it appears that, in the range of frequencies chosen, all the points representing a sine signal are on a line. From the inspection of the existing literature, this result appears to be novel, yet we do not presently have an explanation for this alignment. But as expected, due to a spectral aliasing, the displacement with pulsation of the point on the line is not trivial.

In order to place some reference marks relative to stochastic processes, we considered the fractional Brownian motion [22]. This can be viewed as a stochastic process the probability density of which is a Gaussian. The roughness of its time series is governed by a parameter, the Hurst one (noted $H$ ). The intuitive concept of roughness has to be understood in terms of the long-range memory of a time series. Indeed, $H$ measures the persistence of a time series. The case $0<H \leq 0.5$ corresponds to a long-term switching between high and low values in adjacent pairs (negative autocorrelation). A single high value will probably be followed by a low value and the value after will tend to be high. Such an changeover provide a high roughness. It corresponds to a high degree of randomness (which is graphically confirmed on Figure 3 ) since a tendency of adopting high or low values is not preserved in time. The case $0.5 \leq H<1$ corresponds to a long 


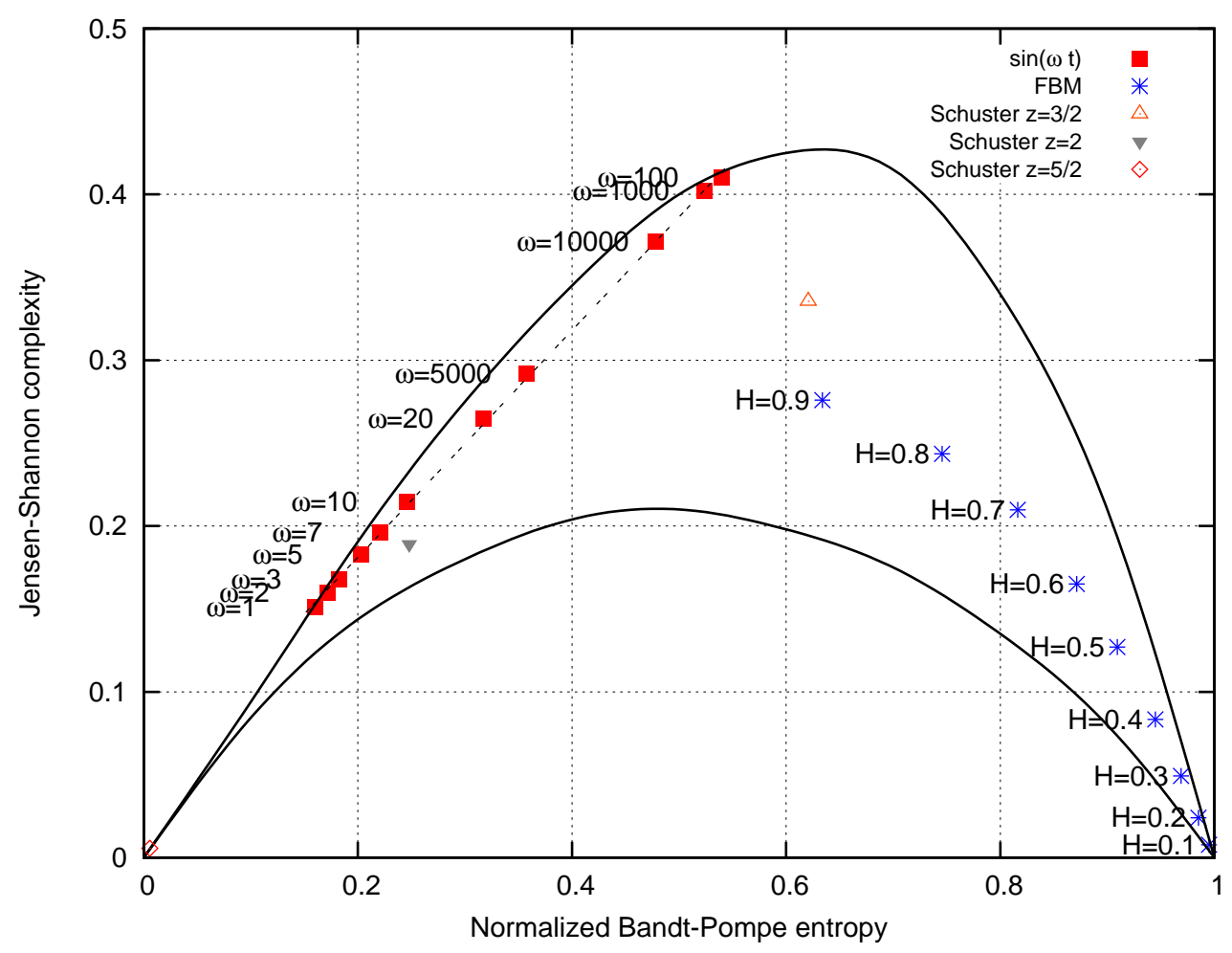

Figure 3: Entropy-complexity plane for $d=5$ for some sine functions of angular frequencies $\omega$ ranging from 1 to $10^{4}$, for fractional Brownian motion with different Hurst parameters, and for the Schuster maps with $z=3 / 2, z=2$ and $z=5 / 2$. The sampling frequency of the sine functions is $f_{s}=100$. The limit curves of maximal and minimal complexities as a function of the permutation entropy are also shown. 
term persistence of high or low values in adjacent pairs (positive autocorrelation). A single high value will probably be followed by a high value and the value after will tend to be high. Thus some permutations are privileged and the disequilibrium increases with $H$.

\section{Transition with the energy density in the entropy-complexity plane}

\subsection{Application to the Antiferromagnetic Hamiltonian Mean Field Model}

As announced in Section 2, one can expect to find much of the information about the dynamics of a mean-field system in the time variation of few collective observables. As it appears in the equation of motion (3) of any particle $i$, one has chosen the modulus, $M$, of the magnetization as one of such a collective observable.

In order to make an entropy-complexity analysis meaningful, one needs to distinguish the set of parameters inherent to the method from those intrinsic to the physical system. Obvious parameters appear to be the embedding dimension $d$ in the first category, and the number of particles $N$ and energy density $\varepsilon$ in the second one. However, since our interest lies in the behavior of the system in the mean field limit $N \rightarrow \infty$, our aim has been to discriminate in our study the impacts of $d$ and of finite- $N$ effects from those of $\varepsilon$.

Concerning the choice of the so-called embedding dimension (or order) $d$, numerical simulations indicate that, at $N$ and $\varepsilon$ fixed, the complexity is increasing with $d$. Indeed, given a time series, one has more chance to observe all the permutations of order e.g. $d=3$ than those of order $d=5$ because $3 !=6 \leq 5 !=120$. Therefore the disequilibrium increases with $d$. As announced in Section 3, we chose to fix $d$ equal to 5. Concerning the dependency of the entropy-complexity results with respect to the number $N$ of bodies, a typical behavior is indicated in the Table 1 .

\begin{tabular}{|c|c|c|c|c|}
\hline$N$ & 5000 & 20000 & 50000 & 200000 \\
\hline$C_{J S}$ & 0.40771 & 0.38465 & 0.38500 & 0.38478 \\
\hline
\end{tabular}

Table 1: Typical evolution of the complexity $C_{J S}$ as a function of the number of particles $N$ with $d=5$. These values have been obtained for $\varepsilon=-0.498$.

A similar behavior was observed at different energy densities. This led us to conclude that in the $\left(s_{B P}, C_{J S}\right)$ plane, the mean field limit seems to be numerically reached for $N=20000$. In the following, one sets consequently $N=20000$ and $d=5$.

We used time series of the modulus of the mean-field $M$ obtained from various long-time fourth-order symplectic computations using a time-step $\Delta \tau=10^{-2}$ in which $M$ was recorded 30000 times every $100 \Delta \tau$ 's. The sampling frequency in the dimensionless units of the system is thus $f_{s}=1$. The trajectory in the $\left(s_{B P}, C_{J S}\right)$ plane of $M$, as the energy per body $\varepsilon$ is varied, is represented on Figure 4, and is superposed on Figure 5 with the reference results of the Fractional Brownian Motion (Hurst parameter is varying) and of sine signals (with various pulsation frequencies) already shown on Figure 3.

\subsection{Interpretation}

One of the benefits of the entropy-complexity analysis is to provide an elegant and compact tool to visualize qualitative changes in the long-time mean-field time behavior depending on the energy density. In particular, Figure 4 shows that the low energy collective dynamics is peculiar, which turns out to be a signature of the bicluster quasistationary state, since this behavior is well separated from the large energy one. Having in mind Rosso et al.'s analysis [28] on the separate 


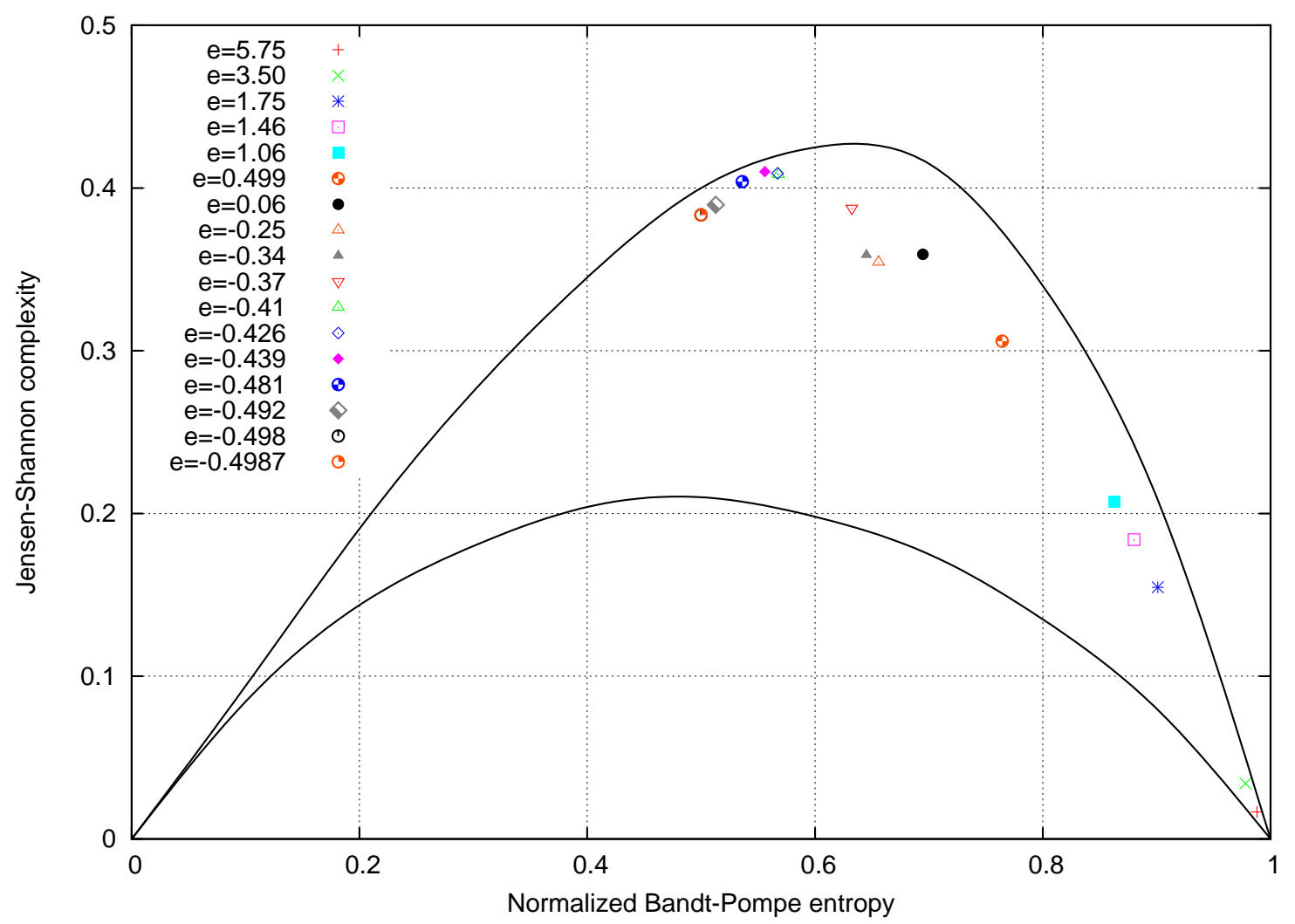

Figure 4: Representation in the entropy-complexity plane of the entropy-complexity analysis of time series of $M(t)$ for $d=5$. The control parameter is the energy density $\varepsilon$. 


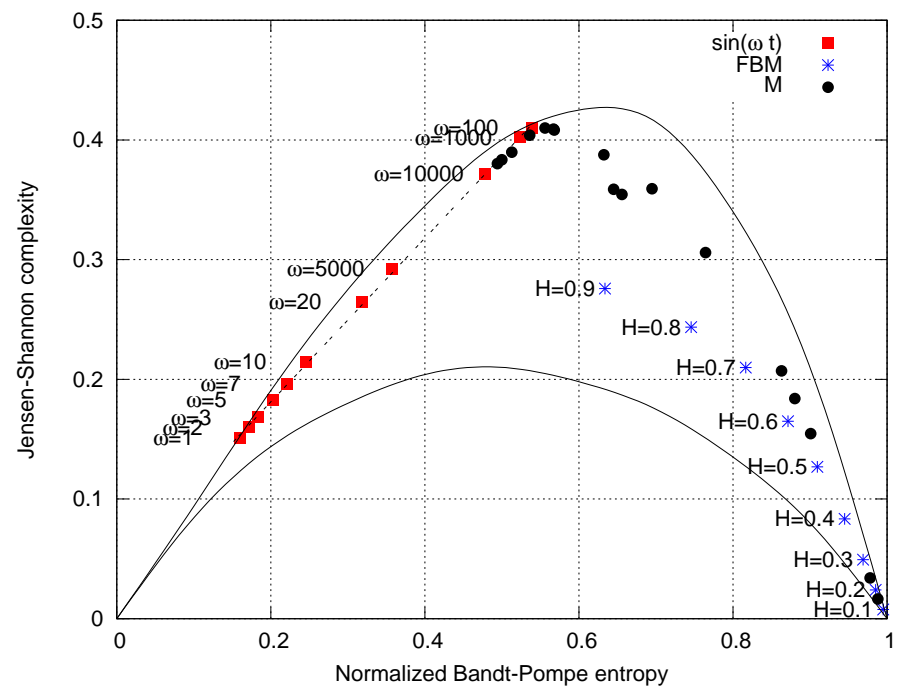

Figure 5: Entropy-complexity representation of the repulsive HMF results shown on Figure 4 superposed to the sine and FBM reference results. Note that, whereas the sampling frequency is $f_{s}=100$ for the sine times series, the sampling frequency is $f_{s}=1$ for the HMF time series.

locations of deterministic chaos and noise in the complexity-entropy plane, the examination of Figure 5 may be interpreted as a transition from a highly complex state at lowest energies to a white noise state in the high energy limit. Let us examine and discuss these two limit behaviors.

For low values of the energy per body $(\varepsilon \rightarrow-1 / 2)$, the entropy-complexity locations of the times series of the modulus of the mean-field coincide with the top of the branch of sine time series. This is in agreement with the dynamical interpretation of the bicluster emergence [4, 17] that comes from the excitation of a linear collective "plasma-like" wave of frequency of order 1 , namely of the order of the sampling frequency used here for the HMF system. This fast timescale may be averaged out to capture the large lifetime scale of the bicluster. Yet the large time variations of $M(t)$ appear here to be completely masked by the rapid oscillations of the signal at about the sampling frequency. Proceeding to a Poincaré's section, presented in Section 5, will serve to evacuate this fast timescale dynamics and will be shown to be able to single-out drastically the low-energy collective dynamics in the entropy-complexity plane.

For high values of $\varepsilon$, the behavior of $M(t)$ appears as being stochastic. This stochasticity of $M(t)$ originates here from a deterministic and non-chaotic (in the $N \gg 1$ limit) model. As it is indeed known for long, stochastic-like behaviors can appear in large assemblies of coupled harmonic oscillators [12]. It is this stochastic-like behavior that ensures the convergence to equilibrium Gibbs-Boltzmann predictions despite the fact that the HMF antiferromagnetic system becomes non-chaotic in the large $N$ - and energy- limits. Indeed, a measure of the magnitude of chaos is given by the largest Lyapunov exponent that scales as $N^{-1 / 3}$, as in the high energy phase of the ferromagnetic HMF [11,31].

To be specific, as the energy density $\varepsilon$ increases, the ratio of the potential energy over the kinetic energy tends to zero and particles tend to behave as free particles with impulsions of the form $p_{i}(t)=p_{i 0} t+q_{i 0}$. The mean-field component $M_{x}$ (or $M_{y}$ ) are then approximated by sums of cosine (or sine) functions of the type $\sum_{i=1}^{N}(1 / N) \cos \left(p_{i 0} t+q_{i 0}\right)$. Under some appropriate 
relabeling of the particles, these sums may be viewed as truncations of a Fourier series expansion of a random (white noise) function [27] assuming a random distribution of the phases $q_{i 0}$.

The mean-field being associated to finite- $N$ Fourier series truncations of a stochastic function has its place in the right bottom corner of the entropy-complexity plane. However, whereas a truly stochastic non-deterministic process should be associated to an entropy-complexity couple remaining at the same location in the right bottom corner of the entropy-complexity plane independently of the sampling period, it will soon be shown that this does not happen here. The sampling period does affect the entropy-complexity locations in our finite- $N$ system, even at large energies, which signals its deterministic nature.

\section{A dynamical signature of a quasi-stationary state}

The objective of the present Section is first to study in more detail the impact of the time resolution in the time series of the observable on the entropy-complexity location. This dependency on the time resolution makes for instance quite problematic the comparison in the entropy-complexity frame of time series coming from different systems. It is therefore desirable to search for routines diminishing as much as possible this dependency. A possibility that will be used in Section 5.2 is to extract the maxima (or minima) of the time series and to proceed to the entropy-complexity analysis on these sub-time series. This amounts to work on a zero-time derivative Poincaré's section of the time signal. This approach will be shown to enable to single out the energy domain where dynamic anomalies take place.

In the present Section, numerical simulations of the repulsive HMF model have been performed starting from different initial conditions than in the previous Sections. Instead of pushing the collective mode by using initial conditions of a sinus-type, we now use simple waterbag conditions in $p$ and a uniform space distribution so that the initial distribution functions are of the type $f_{0}(p, q)=(2 \Delta p)^{-1} \mathbb{1}_{[-\Delta p ; \Delta p]}(p)$.

\subsection{Impact of the time resolution in the entropy-complexity analysis}

The dynamics of the HMF model with 20000 particles has been numerically integrated as in the previous Sections. Then, for several energy densities (or equivalently $\Delta p$ ), two types of time series of the mean-field have been extracted: i) one in which the mean-field was recorded every $10 \Delta \tau$ and ii) one in which the mean-field was recorded every $100 \Delta \tau$, namely every unit time. The results of the corresponding entropy-complexity analysis are presented on Figure 6. Figure 6 offers a clear evidence that the quantitative determination of the location in the entropy-complexity plane may depend strongly on the time resolution of the observable under analysis. Using a smaller sampling period for the same energy density, the entropy-complexity point appears to be pushed to the left corner of the diagram. This dependency on the sampling period may be easily interpreted. For the present case with $d=5$, if the sampling frequency is sufficiently high that on $d=5$ successive points the time series appears as monotonic, successive sampled values appear redundant and the entropy tends to zero. A smooth function (which excludes stochastic processes) is locally monotonic if one zooms in so that in the entropy calculation one almost always counts up permutations associated to monotonic variations on five points. Conversely, diminishing the sampling frequency accounts for a zooming out enabling to view on five successive points the variations of this function inducing the growth of the entropy. Eventually a too large sampling period may result in successive sampled values becoming unrelated shifting the localization in the entropy-complexity plane to the right corner with normalized entropy equal 


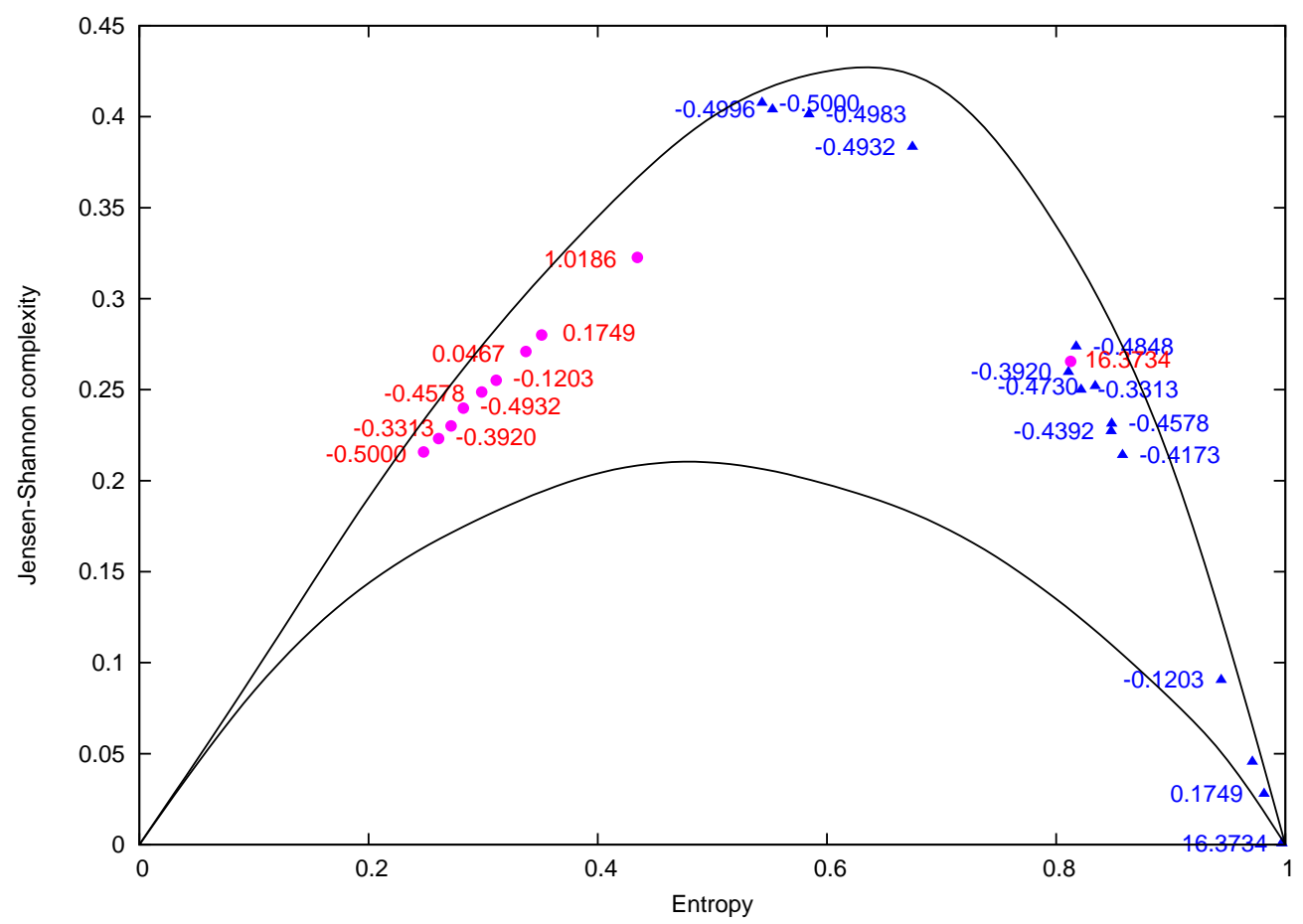

Figure 6: Entropy-complexity representation of the repulsive HMF results with waterbag initial conditions for two time resolutions: i) plain circles correspond to the finer time resolution with time series of the mean-field recorded every 0.1 time unit, ii) plain triangles have been obtained from time series of the mean-field recorded every time unit. The energy densities corresponding to the points are written on the graph. 
to one. Consequently, $d$ being fixed, varying the sampling frequency amounts to modify the time-scale on which the physical observable is analyzed.

In a recent article [23], de Micco and coworkers argued that the choice of the sampling time should result from an optimal tradeoff between those two limit behaviors and be such as to allow a maximization of the statistical complexity. There is however some arbitrariness left in this principle of maximization of the complexity. Moreover, for the model considered here, this would imply changing the sampling time with the energy density so as to maximize the complexity at each density energy preventing any energy comparison in the entropy-complexity plane.

Here, another approach will be chosen that does not need to fix the sampling period and allows an universal comparison between all the different energy cases.

\subsection{The Poincaré's section approach}

Let us first assume that the time resolution used in the time series of the observable under consideration is sufficiently fine, so that one remains far away from the maximal entropy case mentioned above where the too large sampling period make successive sampled values appear unrelated. Then, instead of using the full time series in the entropy complexity analysis, one may proceed to a Poincarés section of the signal by restricting to the series of its relative maxima (or minima). This amounts to doing a Poincaré's section of the signal $M(t)$ on the zero time derivative section $d M / d t=0$ [15]. In the present model, times series of the mean-fields were recorded every time step. We extracted from these time series the relative maxima of the meanfields. Figure 7 represent the corresponding time series of the relative maxima of the modulus of the mean-field, $M$, for three different energy densities close to the fundamental one.

At low energy, the biclustered phase illustrated on Figure 1 may be quantified by the meanfield

$$
\mathbf{M}_{2}=\left(M_{2 x}, M_{2 y}\right)=\left(\frac{1}{N} \sum_{i=1}^{N} \cos 2 q_{i}, \frac{1}{N} \sum_{i=1}^{N} \sin 2 q_{i}\right),
$$

the two components of which are the $n=2$ Fourier coefficients of the spatial distribution, corresponding to the spatial scale $\pi$. It is instructive to compute the time averages of the mean-fields $M$ and $M_{2}$ as a function of the energy density. This has been done using the initial waterbag distributions and results are shown on Figure 8. Equilibrium statistical mechanics would predict vanishing mean-fields in the large $N$ limit which is at odds with the low energy values of the time averages of $M_{2}$. One may remark that the repulsive HMF model is presumably, and not surprisingly, non ergodic at low energies since changing the initial conditions to the previous sinusoidal conditions in the impulsion, that served to push the collective mode, could broaden the energy domain associated to the biclustered phase. Here the biclustered phase appears as a zerotemperature out-of-equilibrium effect that may have some applications to cold atoms [20]. From the observation of Figure 8, one can also remark that, whereas the time average of the meanfield $M_{2}$ rapidly changes from a macroscopic value signaling the biclustered phase about the fundamental state to attain its $O\left(N^{-1 / 2}\right)$ large energy value, the time average of the mean-field $M$ presents some anomalous behaviour in a somehow wider energy domain above the fundamental state. In particular, contrarily to the special initial conditions used in Section 2, using the present waterbag initial conditions the mean-field $M$ remains there below its finite- $N$ noise level $1 / \sqrt{N} \simeq 0.007$ (with $N=20000$ particles), consistently with the fact that unmagnetized states may be realized with inhomogeneous particle densities [8]. 


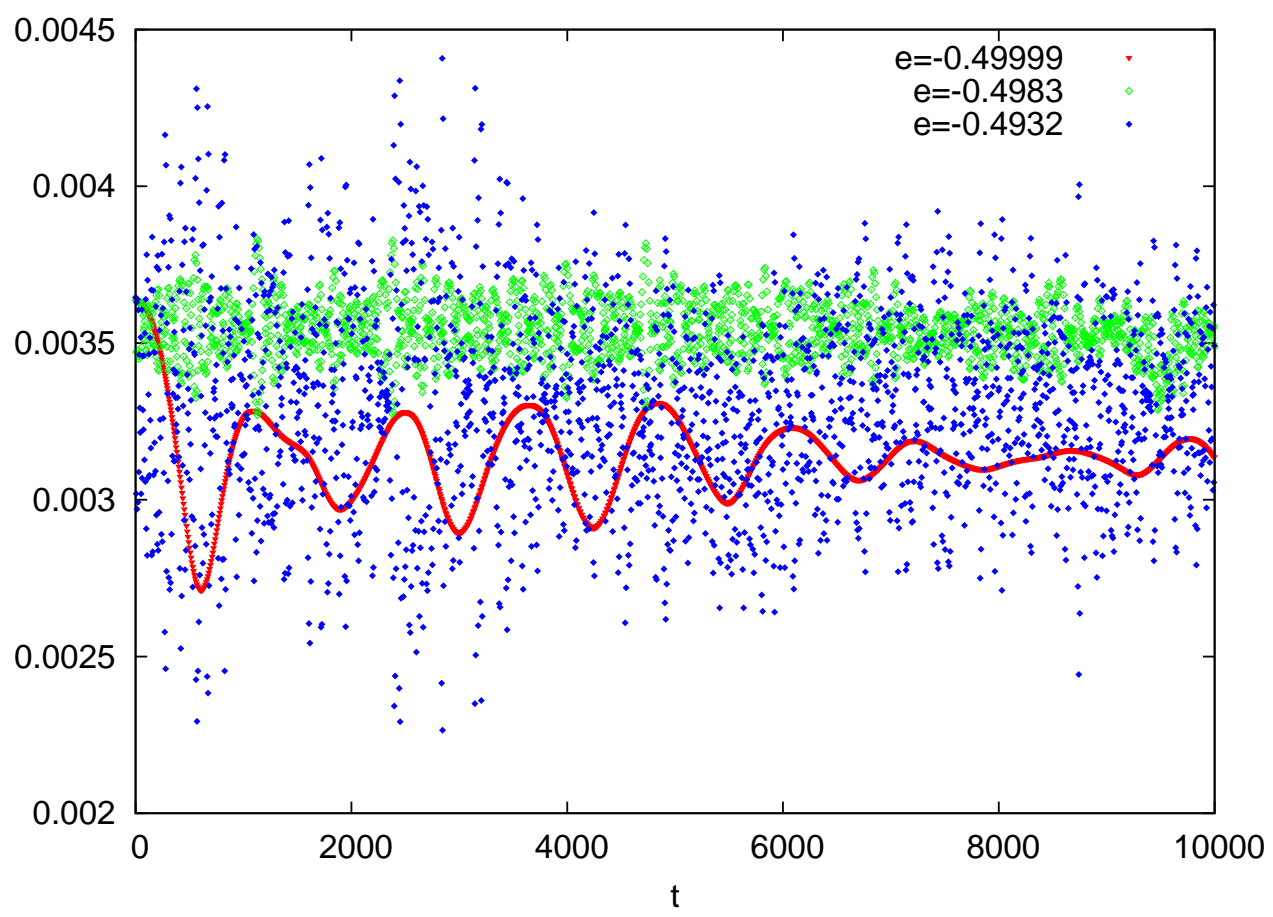

Figure 7: Each point represents the value of a relative maximum of $M$ (y-axis) at its occurrence time ( $x$-axis). Three different simulations with different energy densities are represented. Waterbag initial conditions were used.

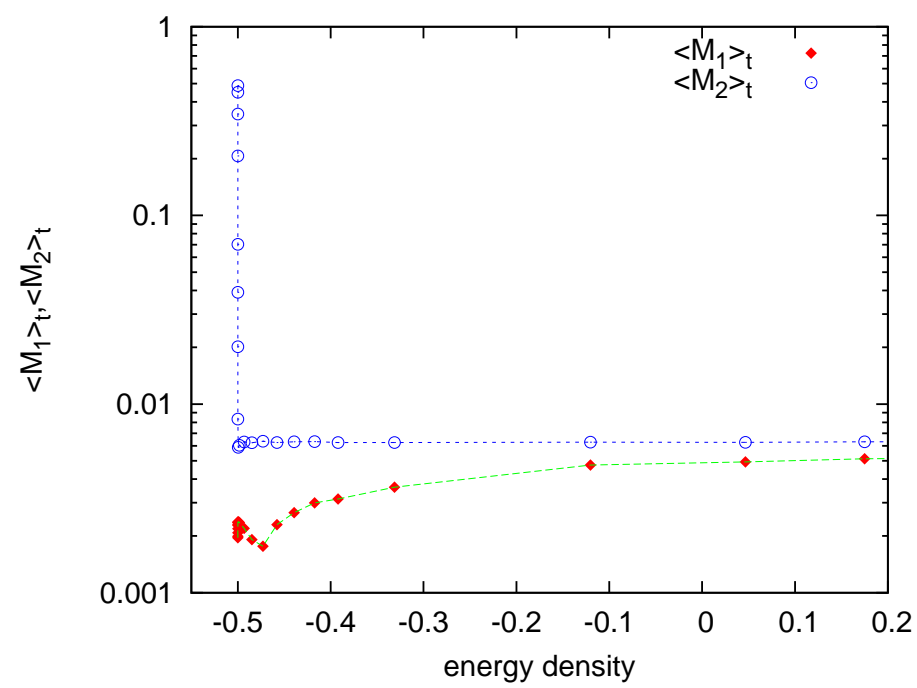

Figure 8: Time averages as a function of the energy densities of the moduli of the mean-fields $M$ (noted here $M_{1}$ ) and $M_{2}$ obtained from waterbag initial conditions. The plot is in lin-log scale. The finite- $N$ noise fluctation amplitude is of the order of $1 / \sqrt{N} \simeq 0.007$ (with $N=20000$ particles). 


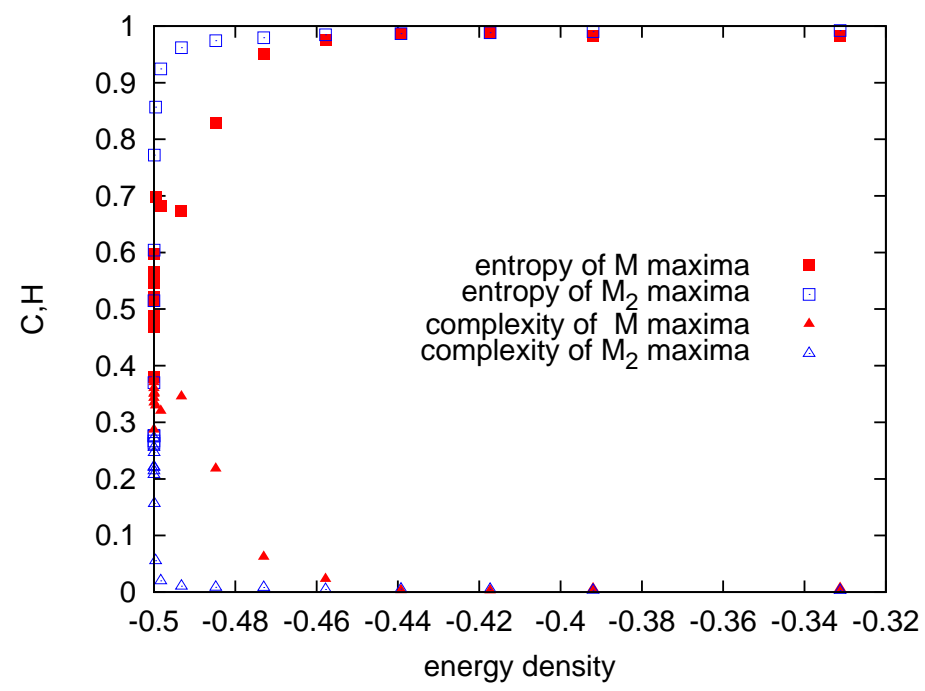

Figure 9: Normalized Bandt-Pompe entropy and Jensen-Shannon complexity associated to the time series of the relative maxima of the magnetization $M(t)$ and of the relative maxima of $M_{2}(t)$ as a function of the energy density.

We extracted the time series given by the relative maxima of both the magnetization, $M$, and of the $M_{2}$ mean-fields. The result of the entropy-complexity analysis of these sub-time series is represented on Figure 9.

The comparison between the Figures 8 and 9 is quite explicit. The entropy-complexity analysis in the Poincaré's section approach shows that the mean-fields, $M$ and $M_{2}$, behave as white-noise on the timescale separating two successive maxima except in the energy domain where they display out-of-equilibrium features. This is very clear for the $M_{2}$ mean-field for which the timeaverage drops rapidly to its thermal value when the energy per body, $\varepsilon$, is just above $-1 / 2$. Correspondingly, there is a sharp variation of the entropy and of the complexity that becomes very low and quickly vanishes. The analysis of the magnetization $M$ shows the same decay of complexity towards zero and growth of the normalized entropy towards one, yet taking place on a wider domain of energy that roughly corresponds to a non-thermal behavior of $M$ presumably due to the presence of some collective mode. These results bring then some evidence that an entropy-complexity analysis is able to unveil out-of-equilibrium dynamical features. Further study will be conducted to assess these results.

\section{Conclusion}

In Section 4, we performed the entropy-complexity analysis of the repulsive HMF model at different energy densities using a fixed arbitrary sampling time. The trajectory of $M$ in the complexity-entropy plane as a function of the energy signals a change from a low-energy lowdimensional behavior of the system dynamics associated to the ergodicity-breaking manifestation of the bicluster quasi-stationary steady state to a high-energy stochastic-like behavior of the mean-field. Yet, as underlined in Section 5, as we are dealing with a deterministic system, the quantitative location in the entropy-complexity plane strongly depends on the choice of the 
sampling period, so that there appears a need to overcome the sampling biases induced by the application of the entropy-complexity frame to time series instead of mappings.

Using a Poincaré's section approach through the use of the sub-time series corresponding to the relative maxima (or minima) provides a frame in which the sampling period is, so to speak, automatically chosen by the system. In Section 5, some numerical evidence is given that the entropy-complexity results using this approach are able to single-out the low-energy anomalous dynamics, that is to separate the low energy domain where the emergence of the collective mode may effectively reduce the dimension of the dynamics from the upper energy domain where the dynamics behaves in a stochastic-like manner.

This result offers interesting perspectives for this approach, e.g. as a novel - easy to handle dynamical indicator to estimate the respective weights of collective modes versus turbulence, in domains such as fluid dynamics or plasma physics.

\section{Acknowledgement}

A useful suggestion by an anonymous Referee of a Poincaré's section analysis and a reference communication by O.A. Rosso are gratefully acknowledged.

\section{References}

[1] Antoni, M., Ruffo, S., 1995. Clustering and relaxation in hamiltonian long-range dynamics. Physical Review E $52(3), 2361$.

[2] Badii, R., Politi, A., 1999. Complexity: Hierarchical Structures and Scaling in Physics. Cambridge Nonlinear Science Series. Cambridge University Press.

[3] Bandt, C., Pompe, B., Apr 2002. Permutation entropy: A natural complexity measure for time series. Phys. Rev. Lett. 88, 174102.

URL http://link.aps.org/doi/10.1103/PhysRevLett.88.174102

[4] Barré, J., Bouchet, F., Dauxois, T., Ruffo, S., 2002. Birth and long-time stabilization of out-of-equilibrium coherent structures. The European Physical Journal B - Condensed Matter and Complex Systems 29 (4), 577-591. URL http://dx.doi.org/10.1140/epjb/e2002-00342-3

[5] Campa, A., Dauxois, T., Ruffo, S., 2009. Statistical mechanics and dynamics of solvable models with long-range interactions. Physics Reports 480 (3 - 6), 57 - 159. URL http://www.sciencedirect.com/science/article/pii/S0370157309001586

[6] Dauxois, T., Holdsworth, P., Ruffo, S., 2000. Violation of ensemble equivalence in the antiferromagnetic mean-field xy model. The European Physical Journal B-Condensed Matter and Complex Systems 16 (4), 659-667.

[7] De Micco, L., Fernndez, J. G., Larrondo, H. A., Plastino, A., Rosso, O. A., 2012. Sampling period, statistical complexity, and chaotic attractors. Physica A: Statistical Mechanics and its Applications 391 (8), 2564-2575.

[8] Ettoumi, W., Firpo, M.-C., 2011. Linear theory and violent relaxation in long-range systems: a test case. Journal of Physics A: Mathematical and Theoretical 44 (17), 175002.

URL http: //stacks. iop.org/1751-8121/44/i=17/a=175002

[9] Ettoumi, W., Firpo, M.-C., Mar 2013. Action diffusion and lifetimes of quasistationary states in the hamiltonian mean-field model. Phys. Rev. E 87, 030102.

URL http://link.aps.org/doi/10.1103/PhysRevE.87.030102

[10] Falasco, G., Saggiorato, G., Vulpiani, A., 2015. About the role of chaos and coarse graining in statistical mechanics Physica A: Statistical Mechanics and its Applications 418, 94 - 104, proceedings of the 13th International Summer School on Fundamental Problems in Statistical Physics.

URL http://www.sciencedirect.com/science/article/pii/S0378437114004038

[11] Firpo, M.-C., Jun 1998. Analytic estimation of the lyapunov exponent in a mean-field model undergoing a phase transition. Phys. Rev. E 57, 6599-6603.

URL http://link.aps.org/doi/10.1103/PhysRevE.57.6599

[12] Ford, G. W., Kac, M., Mazur, P., 1965. Statistical mechanics of assemblies of coupled oscillators. Journal of Mathematical Physics 6 (4), 504-515.

URL http://scitation.aip.org/content/aip/journal/jmp/6/4/10.1063/1.1704304 
[13] Gekelman, W., Compernolle, B. V., DeHaas, T., Vincena, S., 2014. Chaos in magnetic flux ropes. Plasma Physics and Controlled Fusion 56 (6), 064002.

URL http://stacks . iop.org/0741-3335/56/i=6/a=064002

[14] Ginelli, F., Takeuchi, K. A., Chaté, H., Politi, A., Torcini, A., Dec 2011. Chaos in the hamiltonian mean-field model. Phys. Rev. E 84, 066211.

URL http://link.aps.org/doi/10.1103/PhysRevE.84.066211

[15] Kantz, H., Schreiber, T., 1997. Nonlinear time series analysis. Vol. 7 of Cambridge Nonlinear Science Series. Cambridge University Press, Cambridge.

[16] Lamberti, P., Martin, M., Plastino, A., Rosso, O., 2004. Intensive entropic non-triviality measure. Physica A: Statistical Mechanics and its Applications 334 (1 - 2), 119 - 131 URL http://www.sciencedirect.com/science/article/pii/S0378437103010963

[17] Leyvraz, F., Firpo, M.-C., Ruffo, S., 2002. Inhomogeneous quasi-stationary states in a mean-field model with repulsive cosine interactions. Journal of Physics A: Mathematical and General 35 (20), 4413. URL http: //stacks. iop.org/0305-4470/35/i=20/a=303

[18] Leyvraz, F., Ruffo, S., 2002. Ensemble inequivalence in systems with long-range interactions. Journal of Physics A: Mathematical and General 35 (2), 285.

URL http://stacks . iop.org/0305-4470/35/i=2/a=308

[19] López-Ruiz, R., Mancini, H., Calbet, X., 1995. A statistical measure of complexity. Physics Letters A 209 (5 - 6), $321-326$.

URL http://www.sciencedirect.com/science/article/pii/0375960195008675

[20] Maciel, J., Firpo, M.-C., Amato, M., 2015. Some statistical equilibrium mechanics and stability properties of a class of two-dimensional hamiltonian mean-field models. Physica A: Statistical Mechanics and its Applications $424,34-43$.

URL http://www.sciencedirect.com/science/article/pii/S0378437114010723

[21] Maggs, J. E., Morales, G. J., 2013. Permutation entropy analysis of temperature fluctuations from a basic electron heat transport experiment. Plasma Physics and Controlled Fusion 55 (8), 085015. URL http: //stacks .iop.org/0741-3335/55/i=8/a=085015

[22] Mandelbrot, B., Van Ness, J., 1968. Fractional brownian motions fractional noises and applications. SIAM Review $10(4), 422-437$.

[23] Micco, L. D., Fernàndez, J. G., Larrondo, H. A., Plastino, A., Rosso, O. A., 2012. Sampling period, statistical complexity, and chaotic attractors. Physica A: Statistical Mechanics and its Applications 391 (8), 2564 - 2575. URL http://www.sciencedirect.com/science/article/pii/S0378437111009782

[24] Olivares, F., Plastino, A., Rosso, O. A., 2012. Contrasting chaos with noise via local versus global information quantifiers. Physics Letters A 376 (19), 1577-1583.

[25] Olmi, S., Martens, E. A., Thutupalli, S., Torcini, A., Sep 2015. Intermittent chaotic chimeras for coupled rotators. Phys. Rev. E 92, 030901.

URL http://link.aps.org/doi/10.1103/PhysRevE.92.030901

[26] Omel'chenko, O. E., Wolfrum, M., Maistrenko, Y. L., Jun 2010. Chimera states as chaotic spatiotemporal patterns. Phys. Rev. E 81, 065201.

URL http://link.aps.org/doi/10.1103/PhysRevE. 81.065201

[27] Osborne, A. R., 1982. The simulation and measurement of random ocean wave statistics. In: Osborne, A. R., Rizzoli, P. M. (Eds.), Topics in Ocean Physics. North-Holland, Amsterdam, pp. 515 - 550.

[28] Rosso, O. A., Larrondo, H. A., Martin, M. T., Plastino, A., Fuentes, M. A., Oct 2007. Distinguishing noise from chaos. Phys. Rev. Lett. 99, 154102.

URL http://link.aps.org/doi/10.1103/PhysRevLett.99.154102

[29] Scargle, J. D., 1989. An Introduction to Chaotic and Random Time-series Analysis. Intnl. J Imag. Syst. Tech. 1, 243-253.

[30] Shannon, C., July 1948. A mathematical theory of communication. The Bell System Technical Journal 27 (3), 379-423.

[31] Takeuchi, K. A., Chaté, H., Ginelli, F., Politi, A., Torcini, A., Sep 2011. Extensive and subextensive chaos in globally coupled dynamical systems. Phys. Rev. Lett. 107, 124101 URL http://link.aps.org/doi/10.1103/PhysRevLett.107.124101

[32] Tennyson, J., Meiss, J., Morrison, P., 1994. Self-consistent chaos in the beam-plasma instability. Physica D: Nonlinear Phenomena 71 (1), $1-17$. URL http://www.sciencedirect.com/science/article/pii/0167278994901783

[33] Tsallis, C., 03 1999. Nonextensive statistics: theoretical, experimental and computational evidences and connections. Brazilian Journal of Physics 29, $1-35$.

[34] Tsallis, C., Cirto, L., 2014. Thermodynamics is more powerful than the role to it reserved by boltzmann-gibbs statistical mechanics. The European Physical Journal Special Topics 223 (11), 2161-2175. 
URL http://dx.doi.org/10.1140/epjst/e2014-02256-1

[35] Turchi, A., Fanelli, D., Leoncini, X., 2011. Existence of quasi-stationary states at the long range threshold. Communications in Nonlinear Science and Numerical Simulation 16 (12), 4718 - 4724, sI:Complex Systems and Chaos with Fractionality, Discontinuity, and Nonlinearity.

URL http://www.sciencedirect.com/science/article/pii/S1007570411001432

[36] Weck, P. J., Schaffner, D. A., Brown, M. R., Wicks, R. T., Feb 2015. Permutation entropy and statistical complexity analysis of turbulence in laboratory plasmas and the solar wind. Phys. Rev. E 91, 023101.

URL http://link . aps .org/doi/10.1103/PhysRevE.91.023101

[37] Wold, H., 1938. A Study in the Analysis of Stationary Time Series. Almqvst and Wiksell, Uppsala.

[38] Yamaguchi, Y. Y., Barré, J., Bouchet, F., Dauxois, T., Ruffo, S., 2004. Stability criteria of the vlasov equation and quasi-stationary states of the $\{\mathrm{HMF}\}$ model. Physica A: Statistical Mechanics and its Applications 337 (1 - 2), 36 66.

URL http://www. sciencedirect.com/science/article/pii/S0378437104001256

[39] Yoshida, H., 1990. Construction of higher order symplectic integrators. Physics Letters A 150 (57), $262-268$

URL http://www.sciencedirect.com/science/article/pii/0375960190900923 